\title{
GROUP-SIZE EFFECTS AND PARENTAL INVESTMENT STRATEGIES DURING INCUBATION IN JOINT-NESTING TAIWAN YUHINAS (YUHINA BRUNNEICEPS)
}

\author{
HSIAO-WEI YUAN, ${ }^{1}$ SHENG-FENG SHEN, ${ }^{2,3}$ KAI-YIN LIN, ${ }^{2}$ AND PEI-FEN LEE ${ }^{2,4}$
}

\begin{abstract}
We investigated the effect of group size on incubation effort in Taiwan Yuhinas (Yuhina brunneiceps) at the Highlands Experimental Farm of National Taiwan University at Meifeng, Nantou County, central Taiwan, during 2000 and 2001. The Taiwan Yuhina is a joint-nesting, cooperatively breeding species endemic to Taiwan. We compared differences in parental investment among individuals of different sexes and status, explored the effect of group size on group incubation effort, and investigated whether individuals show compensatory reductions in care with respect to the number of females laying. Constancy of incubation increased as group size increased. Alpha females exhibited a significantly greater incubation effort than other individuals, but effort was similar among other group members. Both alpha males and females decreased their relative and absolute incubation effort as group size increased (i.e., there was a compensatory reduction in parental effort). However, beta pairs maintained a consistent but low incubation effort when either gamma pairs or an extra individual joined the group. Our study also demonstrated a new potential group-size benefit for cooperatively breeding birds - an increase in the constancy of incubation. Received 6 July 2004, accepted 31 March 2005.
\end{abstract}

The effect of group size on individual fitness is one of the most important aspects in understanding the evolution of sociality (Brown 1983, Kokko et al. 2001). In cooperatively breeding animals, individuals share parental effort with other group members. The optimal parental investment of each individual depends largely on the sum of other group members' parental efforts; that is, parental effort is affected by group size. Much attention has been paid to how individual provisioning effort is affected by group size in helper-atthe-nest systems (Hatchwell 1999). Two types of provisioning effort are recognized: additive and compensatory (Hatchwell 1999). Additive provisioning occurs when parents maintain the same provisioning effort, regardless of the number of helpers; thus, the total provisioning rate increases as group size increases (Emlen and Wrege 1991). On the other hand, compensatory provisioning occurs when total effort is comparatively constant and breeding individuals reduce their parental effort in response to increasing levels of effort by helpers (Brown et al. 1978). In a detailed comparative

\footnotetext{
${ }^{1}$ School of Forestry and Resource Conservation, National Taiwan Univ., Taipei 106, Taiwan.

${ }^{2}$ Inst. of Ecology and Evolutionary Biology and Dept. of Life Science, National Taiwan Univ., Taipei 106, Taiwan.

${ }^{3}$ Current address: Dept. of Neurobiology and Behavior, Cornell Univ., Ithaca, NY 14853, USA.

${ }^{4}$ Corresponding author; e-mail: leepf@ntu.edu.tw
}

study, Hatchwell (1999) showed that (1) care tends to be additive when the probability of nestling starvation is high and (2) parents are more likely to show compensatory reductions in care when the chance of starvation is low. In addition, Hatchwell hypothesized that male breeders may tend to exhibit compensatory reductions due to the uncertainty of parentage.

Little research has been conducted on the parental investment strategies of cooperative breeders during incubation (Vehrencamp 1977, Heinsohn and Cockburn 1994, Komdeur 1994). Mammalian studies have demonstrated that variation in the relative contributions of individual helpers to different cooperative activities, such as nursing and guarding, is important (Clutton-Brock et al. 2003). Incubation is certainly an important component of reproductive success in most bird species and has been considered a costly behavior (Visser and Lessells 2001, Reid et al. 2002b). Incubation among cooperative breeders is especially interesting to study because-in contrast to the cues received from nestlings-parents and helpers receive no cues from the eggs as to how much care is being given by other group members.

We investigated the effect of group size on incubation effort in a joint-nesting passerine, the Taiwan Yuhina (Yuhina brunneiceps, hereafter referred to as yuhina). Our study focused on whether additive and compensatory investment strategies, developed in the context 
of nestling provisioning, are also applicable to incubation effort. We explicitly considered two issues. First, we explored the effect of group size on total incubation effort. Second, we investigated whether individuals exhibited compensatory reductions in care by comparing the differences in parental investment strategies between individuals of different sex and status with respect to the number of breeding pairs.

\section{METHODS}

Study population.-The Taiwan Yuhina, a Timaliine babbler, is endemic to Taiwan (Clements 2000). Since Yamashina (1938) first described the species' communal nesting behaviors, there has been no detailed study of this species. We have been studying a color-banded population of Taiwan Yuhinas since 1995 at the Highlands Experimental Farm of National Taiwan University at Meifeng, Nantou County, central Taiwan $\left(24^{\circ} 05^{\prime} \mathrm{N}, 121^{\circ} 10^{\prime}\right.$ E; 2,150 $\mathrm{m}$ in elevation). During June 2000, average daytime and nighttime temperature was $25.2^{\circ} \mathrm{C}$ and $12.2^{\circ} \mathrm{C}$, respectively. The study site has been described in detail elsewhere (Yuan et al. 2004). The yuhinas' breeding season lasts 6 months, usually from March or April to August or September. Yuhinas build open-cup nests 1-15 $\mathrm{m}$ above ground in various substrates along forest edges. Most juveniles $(78 \%)$ disappear from the study area after their hatch year; therefore, most $(92 \%)$ new group members are not closely related. Breeding groups comprise one to three monogamous pairs (mode $=2$ pairs) and sometimes one extra male. Within each group, there is a linear hierarchy of socially monogamous pairs with all pairs contributing some eggs. The combined clutch size increases as group size increases. However, the average number of eggs laid by each female decreases with increasing group size (Yuan et al. 2004). According to data from microsatellite genetic markers, mean reproductive skew (the partitioning of reproduction among same-sex individuals within social groups) is low (Yuan et al. 2004), and all breeding pairs contribute to nest building, incubation, and provisioning (H-WY unpubl. data).

Alpha and beta individuals were identified by observing chasing and displacement behavior among group members. Particular in- dividuals-both males and females-consistently chased and displaced same-sex members of their group. In larger groups, gamma individuals were chased by both alpha and beta individuals (see Yuan et al. 2004 for further details). Sex of all banded individuals (97\% of the birds that we observed were color-banded) was assigned tentatively in the field based on observations of singing and copulation; later, gender was verified against sex-specific genetic markers via PCR (Fridolfsson and Ellegren 1999).

Incubation.-We observed diurnal incubation bouts from blinds $10-15 \mathrm{~m}$ from nests by using $15 \times 40$ image-stabilized binoculars. We recorded incubation effort at 21 nests of 11 breeding groups from June to August in 2000 and from March to August in 2001. The mean total observation time for each nest was $683 \mathrm{~min}$ (range $=252-1,096 \mathrm{~min}$ ) and the mean continuous observation period was 414 min (range $=235-839 \mathrm{~min})$. We successfully identified most individuals $(89 \pm 2.4 \%)$ involved in incubation at each nest. We observed incubation effort during the first 10 days of the incubation period (mean $=5.69$ days \pm 2.33 SD of observation per nest).

Data analysis.-Constancy of incubation (i.e., the time that the eggs are in contact with any adult bird, expressed as the percentage of time eggs were incubated; Deeming 2002) differed from nest to nest. A mixed model was originally used to deal with the problem of repeated measurement of different nest attempts within the same group. However, because "group" (indicating different breeding group) had a negative component of variance, the mixed model was equivalent to GLM in this case (Schall 1991); therefore, GLM was used.

Individual incubation effort was assessed in two ways: as relative incubation effort (RIE) and as absolute incubation effort (AIE). We calculated RIE by dividing each individual's incubation time by the group's total incubation time. We calculated AIE by dividing each individual's incubation time by total observation time (group's total incubation time + time during which nest was unattended). We analyzed individual incubation effort (RIE and AIE) by using the residual maximum likelihood (REML) algorithm (mixed model) in SPSS 12.0 (SPSS, Inc. 2003) with normal er- 


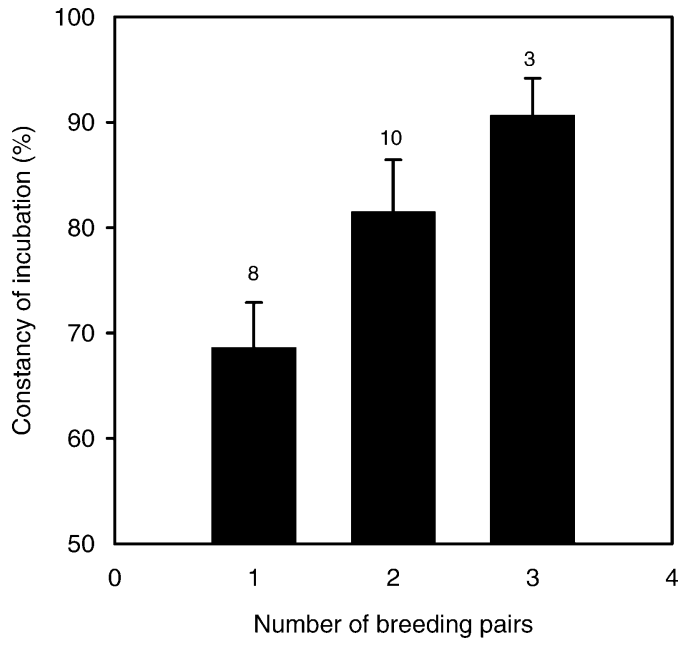

FIG. 1. Relationship between group size (number of breeding pairs) and incubation constancy for Taiwan Yuhinas in Nantou County, central Taiwan, 20002001. Larger groups had significantly greater constancy of incubation than smaller groups. Error bars are \pm SE. Sample size (number of nests) shown above bars.

ror structures, in which both fixed and random terms can be fitted. Random terms control for the use of repeated measurements within a given group, nest (different nest attempts of each group), pair status (i.e., alpha, beta, and gamma birds, and extra males without mates in odd numbered groups), and sex. However, the only interaction term we used in the analysis of alpha's and beta's incubation effort was "group" $\times$ "status" because all other factors had a negative component of variance (Schall 1991). The incubation effort of gamma pairs and extra males were excluded in all analyses due to their small sample sizes. However, gamma pairs affected group size and "extra" was used as a categorical variable to see if an extra male would affect other individuals' incubation effort. Least significant difference (LSD) post hoc pairwise comparisons were used to compare individual effort for each sex and social rank.

Given that sex and status had a significant interaction effect, we also use a mixed model REML to analyze factors affecting alpha pairs' and beta pairs' incubation efforts separately. However, group fitted as the random term had a negative component of variance, so the mixed model is equivalent to GLM in this analysis. We report means $\pm \mathrm{SE}$ through-
TABLE 1. Results from a mixed model (residual maximum likelihood, REML) of relative incubation effort (RIE) for breeders of alpha and beta pairs of Taiwan Yuhinas in Nantou County, central Taiwan, 20002001.

\begin{tabular}{llrl}
\hline \multicolumn{1}{c}{ Effect } & \multicolumn{1}{c}{ df } & \multicolumn{1}{c}{$F$} & \multicolumn{1}{c}{$P$} \\
\hline Intercept & 1,8 & 15.59 & 0.004 \\
Sex & 1,34 & 4.21 & 0.048 \\
Status & 1,8 & 5.94 & 0.040 \\
Group size & 1,7 & 1.89 & 0.21 \\
Sex $\times$ status & 1,34 & 4.68 & 0.038 \\
Status $\times$ group size & 1,8 & 2.99 & 0.12 \\
Sex $\times$ group size & 1,34 & 1.53 & 0.23 \\
Extra $\times$ group size & 1,6 & 0.09 & 0.77 \\
\hline
\end{tabular}

a Status $\times$ group size was included in the model as a random factor. Estimate of variance $=33.90 \pm 46.37(\mathrm{SE})$

out this paper. All tests are two-tailed, with a significance criterion of $P<0.05$.

\section{RESULTS}

Factors influencing constancy of incubation and individual contributions to incubation.Constancy of incubation (\% of time eggs were incubated) was significantly influenced by group size $\left(\mathrm{GLM}, F_{1,21}=7.32, P=0.014\right.$; Fig. 1), but not by month of breeding $\left(F_{1,21}=\right.$ $0.20, P=0.25)$, number of previous nesting attempts $\left(F_{1,21}=0.297, P=0.59\right)$, or days after first incubation $\left(F_{1,21}=0.475, P=0.50\right)$. Individual RIE differed between sexes (group sizes combined, alpha and beta birds combined: $23.13 \pm 2.38 \%$ for males, $35.64 \pm$ $3.64 \%$ for females) and status class (group sizes combined, males and females combined: $36.34 \pm 2.91 \%$ for alpha birds, $17.63 \pm$ $2.33 \%$ for beta birds), and there was a significant sex $\times$ status interaction (Table 1). AIE did not differ between the sexes $(P=0.18)$ or between birds of different status $(P=$ 0.11 ), but there was a significant interaction between sex and status (Table 2). Pairwise comparisons show that alpha females contributed more than all other birds in RIE (group sizes combined): alpha female (45.73 \pm $3.93 \%)$ versus alpha male $(26.94 \pm 3.23 \%, P$ $<0.001$ ); alpha female versus beta female $(18.28 \pm 3.87 \%, P<0.001)$. Alpha females also contributed more than all other birds in AIE (group sizes combined): alpha female $(34.16 \pm 2.54 \%)$ versus alpha male $(20.22 \pm$ $2.44 \%, P<0.001)$; alpha female versus beta female $(15.92 \pm 3.35 \%, P=0.001)$. There 
TABLE 2. Results from a mixed model (residual maximum likelihood, REML) of absolute incubation effort (AIE) for breeders of alpha and beta pairs of Taiwan Yuhinas in Nantou County, central Taiwan, 2000-2001.

\begin{tabular}{llrl}
\hline \multicolumn{1}{c}{ Effect } & \multicolumn{1}{c}{ df } & \multicolumn{1}{c}{$F$} & \multicolumn{1}{c}{$P$} \\
\hline Intercept & 1,6 & 10.87 & 0.018 \\
Sex & 1,28 & 1.91 & 0.18 \\
Status & 1,5 & 3.74 & 0.11 \\
Group size & 1,5 & 0.20 & 0.67 \\
Sex $\times$ status & 1,28 & 4.37 & 0.046 \\
Status $\times$ group size & 1,5 & 1.63 & 0.26 \\
Sex $\times$ group size & 1,28 & 0.40 & 0.53 \\
Extra $\times$ group size & 1,3 & 0.48 & 0.54 \\
\hline
\end{tabular}

a Status $\times$ group size was included in the model as a random factor. Estimate of variance $=17.02 \pm 30.77(\mathrm{SE})$.
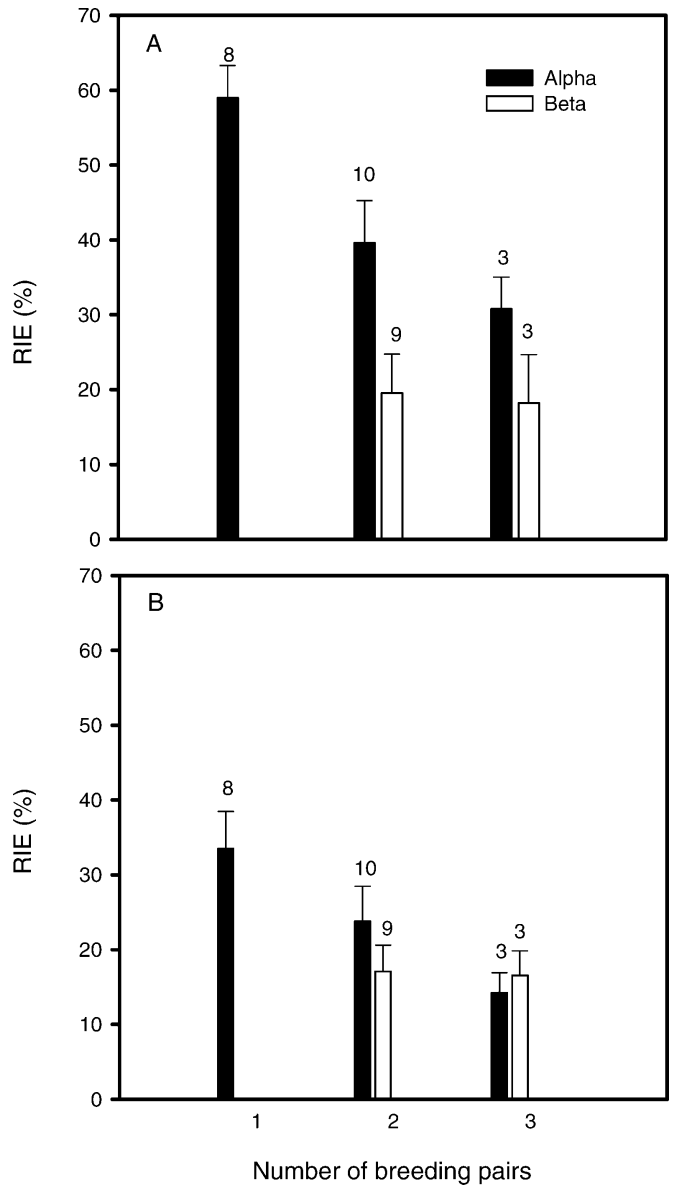

FIG. 2. Mean ( \pm SE) relative incubation effort (RIE) of (A) females and (B) males in different group sizes (number of breeding pairs) of Taiwan Yuhinas in Nantou County, central Taiwan, 2000-2001. Sample size (number of nests) shown above bars.
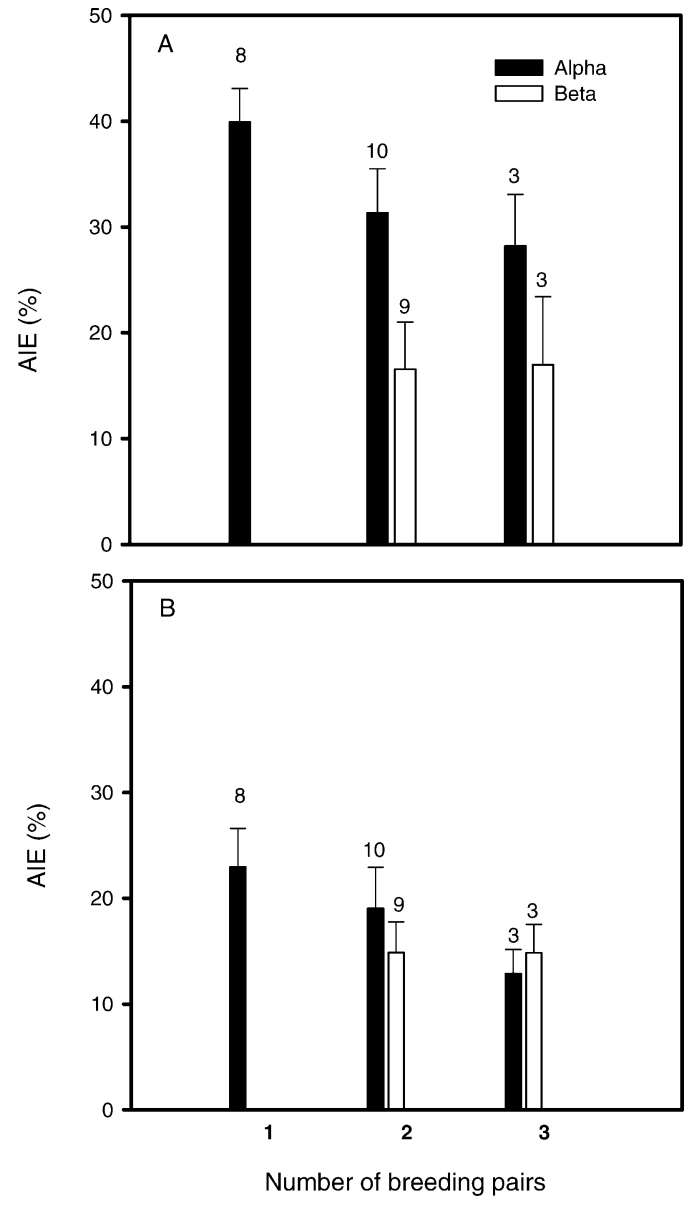

FIG. 3. Mean ( \pm SE) absolute incubation effort (AIE) of (A) females and (B) males in different group sizes (number of breeding pairs) of Taiwan Yuhinas in Nantou County, central Taiwan, 2000-2001. Sample size (number of nests) shown above bars.

were no significant differences among other individuals in either RIE or AIE (Figs. 2 and 3).

Incubation effort between sexes and group sizes of different status.-Because there was a strong sex $\times$ status interaction, we further analyzed alpha and beta pairs separately. RIE of alpha females was greater than that of alpha males (GLM, $F_{1,37}=6.43, P=0.016$ : Fig. 2) and RIE of the three group sizes differed (males and females combined, alpha birds only): $46.25 \pm 2.58 \%$ (one breeding pair), $32.55 \pm 3.97 \%$ (two breeding pairs), and $22.52 \pm 4.32 \%$ (three breeding pairs) $\left(F_{1,37}=\right.$ 16.24, $P<0.001$; Fig. 2). There was no in- 
teraction between sex and group size $\left(F_{1,37}=\right.$ $1.37, P=0.25)$ or between the extra male and group size $\left(F_{1,37}=0.03, P=0.87\right)$. AIE of alpha pairs differed marginally between the sexes (group sizes combined): $20.22 \pm 2.44 \%$ (males), $34.16 \pm 2.54 \%$ (females) $\left(F_{1,37}=\right.$ $3.78, P=0.059$; Fig. 3 ). AIE of alpha pairs also differed between group sizes (males and females combined, alpha birds only): $31.44 \pm$ $3.20 \%$ (one breeding pair), $25.80 \pm 3.11 \%$ (two breeding pairs), and $20.53 \pm 4.19 \%$ (three breeding pairs) $\left(F_{1,37}=4.04, P=\right.$ 0.043; Fig. 3). Again, the interaction between sex and group size $\left(F_{1,37}=0.40, P=0.53\right)$ and the interaction between the extra male and group size $\left(F_{1,37}=0.29, P=0.59\right)$ were not significant. In beta pairs, all factors tested above did not significantly affect either RIE or AIE.

\section{DISCUSSION}

To our knowledge, this is the first study showing that constancy of incubation increases with increasing group size. Greater constancy of incubation may provide a more stable thermal environment, resulting in more rapid embryo development (Deeming 2002). This suggests that sharing the incubation effort does not necessarily lead only to "load lightening" of other breeders (Brown and Brown 1981, Crick 1992). Breeders may also maintain the same level of incubation effort, thus increasing overall incubation effort as more individuals participate. This response may be analogous to the "additive" effects of helpers during nestling provisioning (Hatchwell 1999), presumably increasing the fitness of breeders by increasing the number of offspring.

Here, we showed that both alpha males and females reduced their incubation effort when more individuals were present in the group. Beta pairs, however, maintained a constant effort when more individuals participated in incubation. This phenomenon explains why constancy of incubation varies positively with group size. Attracting additional group members reduces the cost of parental care for alpha individuals while providing improved incubation constancy. Given that the alpha females contribute more to incubation than other members of their respective groups, it is reasonable to expect that alpha females could gain more benefit from larger groups in terms of lightening their work load. This may explain why the survival probabilities of alpha females, but not alpha males, increase with group size (Shen 2002).

In this study, alpha females contributed significantly more to diurnal incubation effort than other individuals. Yuan et al. (2004) found that female yuhinas also contribute more to nocturnal incubation. Female-biased asymmetry in parental care has long been attributed to a sex-bias in the uncertainty of parentage (Trivers 1972, Queller 1997). In cooperatively breeding species, it has been argued that selection favors male survival over parental care, whereas in females, selection favors behavior that promotes offspring survival, largely due to the fact that females have a greater confidence of parentage (Cockburn 1998, Hatchwell 1999). Extra-group paternity is high in yuhinas $(21.4 \%$; H-WY unpubl. data), and it seems appropriate to adopt the parentage uncertainty hypothesis to explain the high incubation effort of alpha females, but not males. However, in most joint-nesting species, the male does the bulk of incubating, especially at night (Vehrencamp 2000, Vehrencamp and Quinn 2004). A possible functional explanation for males taking over nocturnal incubation-which is energetically expensive and imposes a greater risk of predation-is that it allows their mates to produce larger clutches, lay replacement clutches, or contribute more to nestling care (Vehrencamp 2000). Moreover, the low incidence of extragroup paternity in those joint-nesting species may promote greater contributions of parental care among the males (review in Cockburn 1998); yuhinas, on the other hand, exhibit a greater extent of extra-pair paternity. Therefore, different fitness components must be considered to predict patterns of variation in parental investment strategies in complex social groups.

Although parental investment strategies developed according to provisioning effort can be analogous to incubation effort, as we have shown above, we believe that there are some important differences that need further examination. For example, are there any differences in parental investment strategies with respect to incubation effort and provisioning, and, if so, why? For cooperatively breeding mam- 
mals, much more attention has been given to comparing division of labor of different activities, such as babysitting, pup feeding, and guarding, than for birds (Clutton-Brock et al. 2003). In birds, Magrath and Komdeur (2003) also argued that the tradeoffs between parental effort and mating effort have more commonly been observed during incubation than during the nestling feeding period in cooperatively breeding species, although little empirical evidence supports this view. Another related question is why there are fewer helpers in cooperatively breeding species participating in incubation. Clutton-Brock (1991) speculated that one stimulus for male help with incubation may be an attempt to minimize the probability that females will destroy each others' eggs. Few studies have been conducted to evaluate this hypothesis. Given that incubation is an important component of reproductive costs in birds (Reid et al. 2002a), it is surprising that there are so few studies dealing with parental investment strategies during incubation in cooperatively breeding birds.

Our study provides an example of different parental investment strategies exhibited by males and females during incubation, and a potential new group benefit of cooperative breeding that increases incubation effort. We also suggest that AIE should be a better measure than RIE of an individual's incubation effort. Because AIE reflects the real effort and cost of nesting that each individual contributes and bears, respectively, and because RIE represents only the relative contribution (potentially omitting additional information-as we found in this study), only AIE can indicate the extent to which work loads are lightened as group size increases. Future work on incubation in cooperatively breeding birds will give us a better understanding of the effect of helpers and co-breeders on other group members' parental effort and, thus, will have profound implications for the evolution of different investment strategies in different cooperative breeding systems.

\section{ACKNOWLEDGMENTS}

We thank S. L. Vehrencamp, H. L. Mays, Jr., and three anonymous reviewers for valuable comments on a draft of our manuscript. We thank F. Vermeylen for statistical consultation. We also thank M.-C. Tsai, K.Z. Lin, and workers at Meifeng Farm for logistical support. Finally, we greatly appreciate the volunteers from the NTU Nature Conservation Students' Club and School of Forestry and Resource Conservation, in particular H.-Y. Hung, I.-H. Chang, K.-D. Zhong, and S.-W. Fu for their help in the field. This study was supported by the National Science Council and the Council of Agriculture, Taiwan.

\section{LITERATURE CITED}

BRown, J. L. 1983. Cooperation-a biologist's dilemma. Advances in the Study of Behavior 13:1-37.

Brown, J. L. AND E. R. Brown. 1981. Kin selection and individual selection in babblers. Pages 244256 in Natural selection and social behavior: recent research and new theory (R. D. Alexander and D. E. Tinkle, Eds.). Chiron Press, New York.

Brown, J. L., D. D. Dow, E. R. Brown, And S. D. BRown. 1978. Effects of helpers on feeding of nestlings in Grey-crowned Babbler (Pomatostomus temporalis). Behavioral Ecology and Sociobiology 4:43-59.

Clements, J. F. 2000. Birds of the world: a checklist. Ibis Publishing, Vista, California.

Clutton-Brock, T. H. 1991. The evolution of parental care. Princeton University Press, Princeton, New Jersey.

Clutton-Brock, T. H., A. F. Russell, and L. L. SharPE. 2003. Meerkat helpers do not specialize in particular activities. Animal Behaviour 66:531-540.

CockBURN, A. 1998. Evolution of helping behavior in cooperatively breeding birds. Annual Review of Ecology and Systematics 29:141-177.

CRICK, H. Q. P. 1992. Load-lightening in cooperatively breeding birds and the cost of reproduction. Ibis 134:56-61.

DeEming, D. C. 2002. Behavior patterns during incubation. Pages 63-87 in Avian incubation: behavior, environment, and evolution (D. C. Deeming, Ed.). Oxford University Press, New York.

EmLEn, S. T. AND P. H. Wrege. 1991. Breeding biology of White-fronted Bee-eaters at Nakuru: the influence of helpers on breeder fitness. Journal of Animal Ecology 60:309-326.

Fridolfsson, A. K. AND H. Ellegren. 1999. A simple and universal method for molecular sexing of nonratite birds. Journal of Avian Biology 30:116-121.

HATChwEll, B. J. 1999. Investment strategies of breeders in avian cooperative breeding systems. American Naturalist 154:205-219.

Heinsohn, R. AND A. CockBuRn. 1994. Helping is costly to young birds in cooperatively breeding White-winged Choughs. Proceedings of the Royal Society of London, Series B 256:293-298.

Kokko, H., R. A. Johnstone, And T. H. CluttonBROCK. 2001. The evolution of cooperative breeding through group augmentation. Proceedings of the Royal Society of London, Series B 268:187196.

Komdeur, J. 1994. Experimental evidence for helping and hindering by previous offspring in the cooperative-breeding Seychelles Warbler Acrocephalus 
sechellensis. Behavioral Ecology and Sociobiology 34:175-186.

Magrath, M. J. L. And J. Komdeur. 2003. Is male care compromised by additional mating opportunity? Trends in Ecology and Evolution 18:424430.

Queller, D. C. 1997. Why do females care more than males? Proceedings of the Royal Society of London, Series B 264:1555-1557.

Reid, J. M., P. Monaghan, and R. G. Nager. 2002a. Incubation and the costs of reproduction. Pages 314-324 in Avian incubation: behaviour, environment, and evolution (D. C. Deeming, Ed.). Oxford University Press, New York.

Reid, J. M., P. Monaghan, AND G. D. Ruxton. 2002b. Males matter: the occurrence and consequences of male incubation in starlings (Sturnus vulgaris). Behavioral Ecology and Sociobiology 51:255261.

SCHALL, R. 1991. Estimation of generalized linear models with random effects. Biometrika 78:719727.

SHEN, S.-F. 2002. Ecology of cooperatively breeding Taiwan Yuhinas (Yuhina brunneiceps) in Meifeng areas. M.Sc. thesis, National Taiwan University, Taipei.
SPSS, INC. 2003. SPSS base 12.0 for Windows user's guide. SPSS Inc., Chicago, Illinois.

Trivers, R. L. 1972. Parental investment and sexual selection. Pages 136-179 in Sexual selection and the descent of man, 1871-1971 (B. G. Campbell, Ed.). Aldine, Chicago, Illinois.

VEHRENCAMP, S. L. 1977. Relative fecundity and parental effort in communally nesting anis, Crotophaga sulcirostris. Science 197:403-405.

VEHRENCAMP, S. L. 2000. Evolutionary routes to jointfemale nesting in birds. Behavioral Ecology 11: 334-344.

VeHRENCAMP, S. L. AND J. S. QUiNN. 2004. Joint laying systems. Pages 177-196 in Cooperative breeding in birds: recent research and new theory (W. D. Koenig and J. Dickinson, Eds.). Cambridge University Press, Cambridge, United Kingdom.

Visser, M. E. AND C. M. Lessells. 2001. The costs of egg production and incubation in Great Tits $(\mathrm{Pa}$ rus major). Proceedings of the Royal Society of London, Series B 268:1271-1277.

YAmAshinA, M. 1938. A sociable breeding habit among timaliine birds. Proceedings of the International Ornithological Congress 9:453-456.

YuAN, H.-W., M. LiU, AND S.-F. SHEN. 2004. Joint nesting in Taiwan Yuhinas: a rare passerine case. Condor 106:862-872. 